\title{
A REVIEW OF ANTIPROTON SOURCE STUDIES, 1991
}

\author{
M. Church
}

7 February 1992

The following is a list of studies which were performed in the Antiproton Source in 1991. They are listed in chronological order and this order does not reflect their relative importance.

1) Antiprotons were stacked to a record $120 \mathrm{~mA}$ stack to try and see if any fundamental limitations existed to stack size at this level. None were found. The production efficiency was measured as a function of stack size, as a benchmark for fut ure improvements. The effects of ion clearing and beam shaking were studied. Beam shaking was found to be necessary at large stack sizes in order to maintain reasonable emittances, as expected.

2) The transverse emittance monitors in the Accumulator were recalibrated using the above large stack. There is still some suspicion that these monitors are not correctly calibrated.

3) The Debuncher transverse aperture was measured and significant efforts were made to improve it. The aperture is still not optimized for large $\triangle P / P$.

4) A large amount of time was dedicated to developing the Accumulator magnet ramp tables for E760 decelerations.

5) The Accumulator $\beta$ functions were measured at several different energies and several different lattice points. These measurements were required for E760 beam energy measurements.

6) The ARF3 $(\mathrm{H}=2) \mathrm{Rf}$ cavity was calibrated and this cavity was used to measure the lattice parameter $\eta$ at different energies. This was required for E760 to extract beam momentum width measurements from beam frequency measurements.

7) The radiation levels at the AP0 target hall were measured as part of the sitewide effort to eliminate possible radiation hazards. Significant radiation levels were measured, and remedial action was taken.

8) The Debuncher TBT orbit measurement system was recommissioned and properly documented (after several years of neglect). 
9) Initial beam tests were made of a quadrupole pickup amplifier prototype. A quadrupole pickup will be installed in 1992 .

10) Measurements were made of the $\bar{p}$ production efficiency as a function of $\bar{p}$ production cycle time in order to understand the effectiveness and need for more Debuncher stochastic cooling.

11) $\bar{p}$ extraction studies were performed in order to refamiliarize personnel with the procedures and to verify that the necessary hardware and software was still operational.

12) Measurement of the $D / A$ line transmission efficiency as a function of trim magnet and septa settings was made in order to understand losses during stacking.

13) A measurement of the Accumulator transverse emittance as a function of momentum was made during stacking in order to understand losses during stacking. Preliminary indications are that a coupling resonance may be causing beam losses. 